\title{
Intervention with Mediterranean diet in the improvement of depressive symptoms in patients recovered from depressive disorder. PREDI-DEP trial preliminary results
}

\author{
A. Sánchez-Villegas ${ }^{1,2}$, B. Cabrera-Suárez ${ }^{3}$, M. Santos-Burguete', P. Molero ${ }^{4,5}$, A.M. González-Pinto ${ }^{6,7,8}$, C. Chiclana9 , J.L.
} Hernández-Fleta ${ }^{3}$

${ }^{1}$ Research Institute of Biomedical and Health Sciences. University of Las Palmas de Gran Canaria, Spain; ${ }^{2}$ Biomedical Research Center Network on Obesity and Nutrition (CIBERobn), Institute of Health Carlos III, Madrid, Spain; ${ }^{3}$ Hospital General de Gran Canaria Dr. Negrin, Las Palmas de Gran Canaria, Spain; ${ }^{4}$ University Clinic of Navarra, Pamplona, Spain; ${ }^{5}$ IDISNA (Instituto de Investigación Sanitaria de Navarra), Pamplona, Spain; ${ }^{6}$ University Hospital of Alava, Vitoria, Spain; ${ }^{7}$ Biomedical Research Center Network on Mental Health (CIBERsam), Institute of Health Carlos III, Madrid, Spain; ${ }^{8}$ School of Medicine, University of the Basque Country, Vitoria, Spain ; ${ }^{9}$ Consulta Dr. Carlos Chiclana, Madrid.

\section{Introduction}

Prevention of depression recurrence is an important goal in the management of depressive patients. Our aim was to assess the effectiveness of a nutritional intervention based on Mediterranean diet to reduce residual depressive symptoms after 4 months of intervention in patients with a previous episode of depression (PREDI-DEP trial).

\section{Methods}

DESIGN: The PREDI-DEP is a multicenter, two-arm, parallel-group clinical trial with 2 years of intervention in patients with a previous major depressive episode in a stage of total or partial remission. The study has been prospectively registered in the U.S. National Library of Medicine (https://clinicaltrials.gov) with NCT number: NCT03081065

INTERVENTION: One group is assigned to a Mediterranean diet supplemented with extra-virgin olive oil and the control group has no nutritional intervention.

MEASUREMENTS: Adherence to the Mediterranean diet was assessed in the intervention group after 3 months of follow-up through a validated Mediterranean Diet Assessment Screener (MEDAS) (0-14 points). Depressive symptoms were assessed after 4 months in the overall sample using the Beck Depression Inventory (BDI) validated in Spain.

STATISTICAL ANALYSIS: Differences in the scores of the MEDAS questionnaire (change in the adherence to the Mediterranean diet after 3 months of intervention) was assessed though a Paired-t test. Generalized Linear Models were used to calculate the adjusted mean changes and their $95 \%$ confidence intervals $(95 \% \mathrm{CI})$ in BDI scores after 4 months of intervention. Results were adjusted for sex, age and baseline score in BDI.

\section{Results}

127 patients of the trial with at least 4 months of follow-up ( $n=72$, intervention group; $n=55$, control group) (70.9\% women).

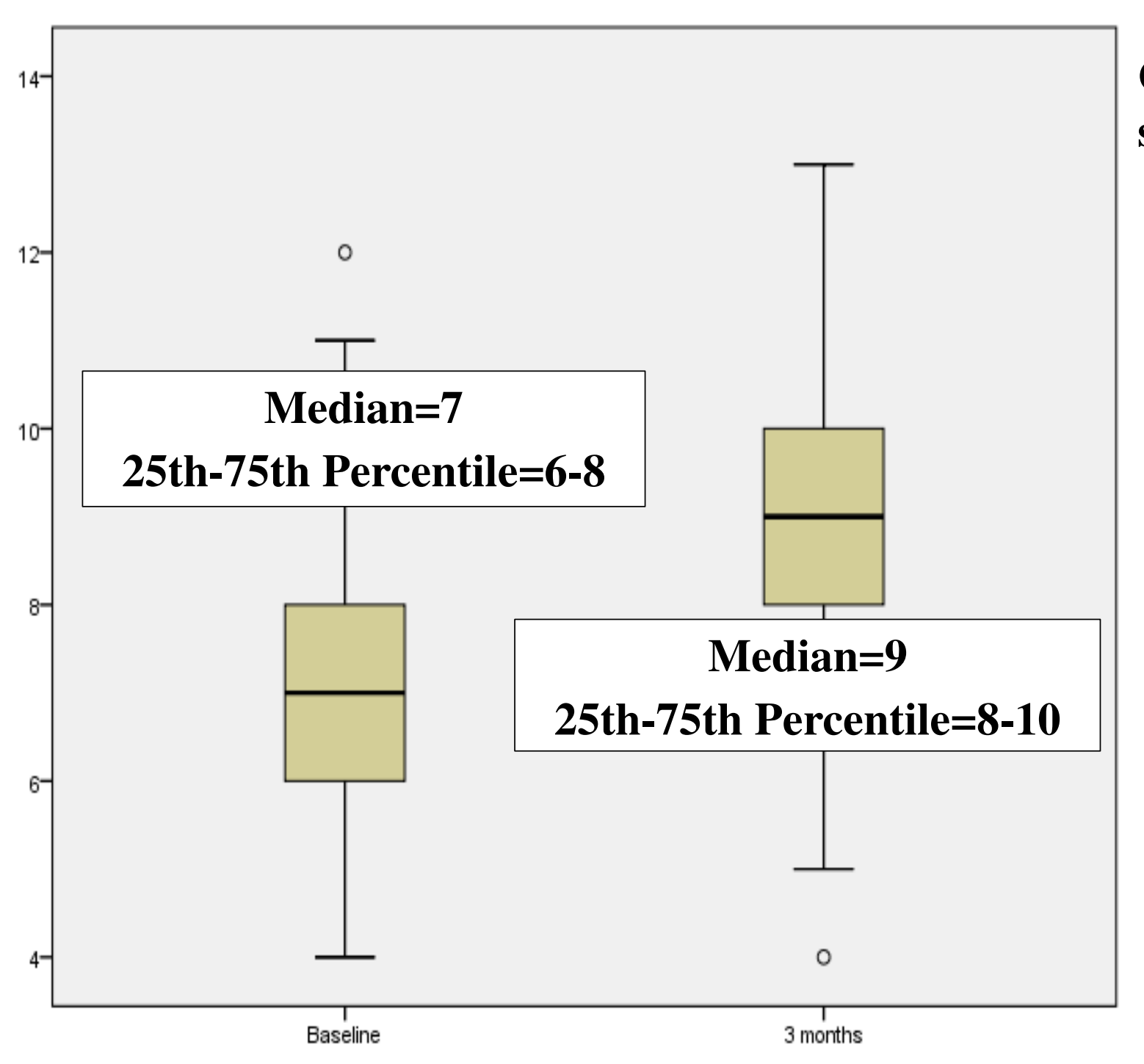

Change in the adherence to the Mediterranean diet (MEDAS score) after 3 months of intervention

Mean change and $95 \% \mathrm{CI}$ in depressive symptoms after four months of nutritional intervention

\begin{tabular}{l|cccc}
\hline & $\mathbf{n}$ & Mean change & $\begin{array}{c}95 \% \text { CI for mean } \\
\text { change }\end{array}$ & $\begin{array}{c}\text { P } \\
\text { ANCOVA }\end{array}$ \\
\hline Control & 55 & $\mathbf{0 . 3 2 8}$ & $\mathbf{- 1 . 6 8 2}$ to 2.337 & $\mathbf{0 . 0 1}$ \\
& & & & \\
\hline Intervention & $\mathbf{7 2}$ & $\mathbf{- 3 . 0 6 0}$ & $\mathbf{- 4 . 8 8 3}$ to $-\mathbf{1 . 2 3 7}$ & \\
\hline
\end{tabular}

Adjusted for baseline depressive symptoms, age and sex

\section{Conclusions}

Our results suggest that the nutritional intervention with Mediterranean diet supplemented with extra virgin olive oil could improve depressive symptoms in patients recovered from a depressive disorder. 